\section{Relationship between mercury levels in blood and urine and complaints of chronic mercury toxicity from amalgam restorations}

\author{
J. Eyeson, ${ }^{1}$ I. House, ${ }^{2}$ Y. H. Yang $^{3}$ and K. A. A. S. Warnakulasuriya ${ }^{4}$
}

IN BRIEF

- Study adds to biochemical knowledge surrounding use of dental amalgam.

- Controversies in relation to dental amalgam safety are explored.

- Provides an insight to objective biochemical investigations of the safety of dental amalgam.

- No correlation was found between perceived amalgam toxicity and mercury levels in blood and urine.

\begin{abstract}
Aim To determine whether patients complaining of oral and medical symptoms perceived to be associated with chronic mercury toxicity have elevated mercury levels in their blood and urine. Methods The study group in this audit were 56 patients presenting to an oral medicine unit with complaints perceived to be related to chronic mercury toxicity. Their symptoms and co-morbidity were charted and mercury levels in blood and urine were biochemically tested by atomic absorption spectrophotometry. Results None had elevated mercury levels in blood or urine above the normal threshold level. Subgroup analysis showed subjects with oral lesions, autoimmune disorders and multiple sclerosis had relatively and significantly higher mercury levels within this cohort, but within the threshold values. When tested by multiple logistic regression adjusted for age and gender, mercury levels in blood or urine, numbers of amalgams were not significant for multiple sclerosis or previously diagnosed autoimmune disease. Conclusion Mercury levels in blood and urine of this cohort of patients with perceived chronic mercury toxicity were within the normal range in accordance with a national laboratory threshold value.
\end{abstract}

\section{INTRODUCTION}

Dental amalgam is routinely used worldwide as a restorative material. Its use has been a contentious issue due to its mercury component. Worldwide 50\% of mercury in the environment is derived from natural sources such as rock erosion, 42\% from the burning of fossil fuels and an estimated 3-4\% from dental amalgam. ${ }^{1}$ Environmental and industrial sources that may contribute to and raise exposure to toxic levels were recently reviewed. ${ }^{2}$ Mercury is a bio-accumulative toxin, a pollutant and a chemical contaminant in fish. ${ }^{3}$ A significant relationship between fish consumption and

\footnotetext{
'Formerly Lecturer/Hon SpR in Oral Surgery, King's College London; ${ }^{2}$ Biomedical Scientist, Medical Toxicology Unit, Guy's and St Thomas' NHS Foundation Trust, London; ${ }^{3}$ Biostistician, Graduate Institute of Oral Health Sciences, College of Dental Medicine, Kaohsiung Medical University, Kaohsiung, Taiwan; ${ }^{4 *}$ Professor/ Consultant Department of Oral Medicine, King's College London Dental Institute at Guy's, King's and St Thomas' Hospitals, London and WHO Collaborating Centre for Oral Cancer \& Precancer, United Kingdom

*Correspondence to: Professor S. Warnakulasuriya Email: s.warne@kcl.ac.uk
}

\section{Online article number E7}

Refereed Paper - Accepted 9 October 2009

DOI: 10.1038/sj.bdj.2010.181

British Dental Journal 2010; 208: E7 blood mercury concentration is reported ${ }^{4}$ but is not considered to pose a risk to human health.

In children urinary mercury levels were reported to be highly correlated with both number of amalgam fillings and time since placement. ${ }^{5}$ Release of mercury vapour from amalgam restorations is known to occur but intensive research over the past three decades have failed to identify deleterious health outcomes. ${ }^{6,7}$ This is likely to be due to insufficient mercury being released from dental amalgam restorations to cause a medical problem. ${ }^{1,8}$ Although significantly higher mercury levels have been found in the blood and urine of dental personnel, their general health problems have not been found to differ significantly from persons not occupationally exposed to mercury.? Though reproductive effects in dental personnel are not proven, ${ }^{9}$ the possibility of a weak association between moderate to high exposure categories of dental personnel and an increased risk of miscarriage was recently reported. ${ }^{10}$ In a comparative study, a group of subjects who reported amalgam fillings to be deleterious to their health did not differ in the number of amalgam restorations, amalgam surfaces or mercury levels in blood, urine or saliva from a control group that had no systemic illness. ${ }^{11}$ However, many subjects reporting symptoms with dental amalgam toxicity were found to have severe somatisation syndromes. ${ }^{11}$

Some of the reported characteristics of acute mercury $(\mathrm{Hg})$ poisoning include neurotoxicity, cardiomyopathies, kidney damage and respiratory failure due to necrotising bronchitis and pneumonitis. ${ }^{12}$ Dental amalgam has been reported as a possible source of mercury nephrotoxicity. ${ }^{13}$ The presence of mercury-containing preservative thimerosal in vaccines had been linked to autism, ${ }^{14}$ but this relationship has been disproved in several epidemiological studies. ${ }^{15}$ Further studies in dental settings are required to clarify the concerns that abound on the topic of amalgam toxicity as a cause of a number of medical conditions.

The aim of this audit was to determine whether patients complaining of oral and general medical symptoms perceived to be associated with chronic mercury toxicity have elevated mercury levels in their blood and urine. 


\section{METHOD}

A group of 56 consecutive patients specifically presenting to the Oral Medicine Unit at Guy's Hospital during 2003-04 with the belief that their oral/medical symptoms or conditions were caused by mercury toxicity from amalgam fillings were included in the audit.

A retrospective study of the clinic notes of patients in this series with suspected mercury amalgam toxicity was undertaken and demographic data and patients' presenting complaints were transcribed. Their medical histories, any co-morbidity information and the number of amalgam restorations found in the radiographs taken at their first visit were recorded.

Skin patch testing against the European Standard and Dental Material series (Trolab Biodiagnostics Ltd: Hermal Patch Test Allergens) was undertaken if any oral lichenoid reactions were noted or a sore mouth was reported at consultation. The patches were placed on the back of the patients and the result read after 48 hours by a designated clinician.

Biochemical investigations that were carried out included blood and urine mercury levels. Blood and urine mercury levels were measured by the medical toxicology laboratory of the Guys and St Thomas' Hospital NHS Trust, London UK (National Laboratory for the UK). Venous blood and urine samples were analysed using a Varian SpectrAA 20 (Varian Ltd, Oxford UK). The analyses were performed by cold vapour atomic absorption (AA) spectrometry with deuterium arc background correction. Biological fluids, digested with mineral acids, were pipetted into a reaction vessel where a reducing agent, tin (II) chloride, was added to reduce the mercury ions in the sample to mercury metal. The mercury was encouraged to evaporate by a stream of argon bubbled through the sample, this also served to mix the sample and reducing agent. The vaporised mercury was swept into a silica windowed flow cell in the light path of an AA spectrometer. The neutral mercury atoms in the vapour phase have resonance frequencies that are specific for this element. A hollow cathode lamp for the mercury provides this in the form of light at a particular wavelength and atoms generated in the path of this light absorb some of the energy. A calibration curve was constructed using known concentration standards, and this was used to calculate the concentration of analyte in a given sample.

Six healthy volunteers (three males, three females, mean age 45yrs) who had no reported medical disorders and who were free of any amalgam restorations were used as controls for the study. Blood and urine analysis were conducted in the national reference laboratory and the laboratory reference value for normal mercury levels in both urine and blood was $<50 \mathrm{nmol} / \mathrm{L}$.

\section{Statistical analysis}

Data analysis was performed using the SAS V9.1 for Windows (SAS Institutes, NC, USA). The t-tests and ANOVA were used to compare mean differences of $\mathrm{Hg}$ levels in blood and urine among groups (gender, age groups, presenting complaints, and medical conditions). The numbers of amalgams were categorised into 3 groups (0-5, 6-10 and 11 or more). Since the comparisons of distributions among groups mostly resulted in small cell sizes, the Chi-square test for trend was adopted instead of the original Chi-square tests. For those presenting complaints and medical conditions which were significant in t-tests, ANOVA or Chi-square testing for trend, the multiple logistic regressions were further analysed to compute the age and gender adjusted odds ratios for $\mathrm{Hg}$ levels in blood or urine, or numbers of amalgams. Differences were considered to be statistically significant when a p-value was $<0.05$.

\section{RESULTS}

Fifty-six consecutive patients with complaints related to mercury toxicity seen over 2 years were included in the audit. The frequency distributions of patients' demographic features, presenting complaints and medical conditions, being variables discussed in this study are given in Table 1.

Thirty-six patients had previously consulted other specialists regarding chronic mercury toxicity with various perceived symptoms. Twenty subjects had previously been labelled to have mercury toxicity by commercial practitioners using unconventional testing panels.

Presenting complaints (Table 1) varied, including oral cavity symptoms (soreness, pain, metallic taste, hypersalivation), neurological symptoms (memory loss, confusion, headaches and dizziness), musculoskeletal complaints (joint ache, including fatigue and malaise) and anxiety and depression. Duration of presenting complaints ranged from three months to most of their adult life.

There were no records in the clinical notes of the number of teeth with amalgam restorations in the case of 11 subjects. The notes of these 11 subjects also did not include radiographs taken at the time of examination. The number of teeth with amalgams ranged from 0-21.

All subjects reporting oral symptoms ( $\mathrm{n}=27$ ) were patch tested using the European Standard and Dental Material Series and all but one found to have a negative response. One subject had a weak response to mercury amalgam.

The mean blood mercury level was $19.9 \pm 11.8$ (range 0-47) nmol/L and the mean urine mercury level was $17.0 \pm 11.6$ (range 3-53) nmol/L. The mean values were significantly lower than the normal threshold value for blood/urine mercury of $50 \mathrm{nmol} / \mathrm{L}$. In four subjects the urine mercury levels were slightly elevated above the normal limit. It was the departmental protocol to repeat the mercury assay after a fish-free diet in those with outlying values. Three such individuals had mercury levels within normal limits on retesting. One patient with an outlying value failed to attend the follow-up appointment. The mean levels of mercury in blood and urine by patient subgroups is shown in Table 2 .

None of the patients had elevated mercury levels above the threshold level. As a secondary analysis we investigated whether any subgroups had relatively higher levels of mercury (though within the normal range) or higher number of amalgams in their mouths.

Relatively higher blood mercury levels were found in subjects with presenting complaints in the oral cavity (23.65 vs 16.33, $\mathrm{p}=0.0320$ ) and blood mercury levels of two patients with multiple sclerosis (MS) was higher than those without MS (47.00 vs 19.33, $\mathrm{p}=0.0186)$ as well as urine levels of those reporting MS (42.00 vs 15.74, $\mathrm{p}=0.0011$ ). In Table 3 , a comparison of the distributions of the number of amalgams, against the reported variables is shown. The significant difference found was in subjects reporting autoimmune diseases ( $p=0.0317)$. 


\begin{tabular}{|c|c|c|c|}
\hline Variable & Category & $\mathrm{n}$ & $\%$ \\
\hline \multirow[t]{2}{*}{ Gender } & Female & 43 & 76.79 \\
\hline & Male & 13 & 23.21 \\
\hline \multirow[t]{6}{*}{ Age group } & $<40$ & 11 & 19.64 \\
\hline & $40-44$ & 14 & 25.00 \\
\hline & $45-49$ & 5 & 8.93 \\
\hline & $50-54$ & 10 & 17.86 \\
\hline & $55-59$ & 6 & 10.71 \\
\hline & $\geq 60$ & 10 & 17.86 \\
\hline \multicolumn{4}{|l|}{ Patients' complaints } \\
\hline \multirow[t]{2}{*}{ Oral cavity complaints } & No & 29 & 51.79 \\
\hline & Yes & 27 & 48.21 \\
\hline \multirow[t]{2}{*}{ Neurological } & No & 41 & 73.21 \\
\hline & Yes & 15 & 26.79 \\
\hline \multirow{2}{*}{$\begin{array}{l}\text { Musculo-skeletal, } \\
\text { fatigue, malaise }\end{array}$} & No & 22 & 39.29 \\
\hline & Yes & 34 & 60.79 \\
\hline \multirow[t]{2}{*}{ Depression and anxiety } & No & 50 & 89.29 \\
\hline & Yes & 6 & 10.71 \\
\hline \multicolumn{4}{|l|}{ Co-morbidity } \\
\hline \multirow[t]{2}{*}{ Allergies } & No & 30 & 53.57 \\
\hline & Yes & 26 & 46.43 \\
\hline \multirow[t]{2}{*}{ Auto-immune disorders } & No & 48 & 85.71 \\
\hline & Yes & 8 & 14.29 \\
\hline \multirow[t]{2}{*}{ Depression } & No & 50 & 89.29 \\
\hline & Yes & 6 & 10.71 \\
\hline \multirow[t]{2}{*}{ Multiple sclerosis } & No & 54 & 96.43 \\
\hline & Yes & 2 & 3.57 \\
\hline \multirow[t]{2}{*}{ Cardiovascular disorders } & No & 43 & 76.79 \\
\hline & Yes & 13 & 23.21 \\
\hline \multirow[t]{2}{*}{ Others } & No & 43 & 76.79 \\
\hline & Yes & 13 & 23.21 \\
\hline
\end{tabular}

Previously diagnosed autoimmune disease was more common in the group with more than 10 amalgams (26.3\%), compared with those with 6-10 amalgams (5.6\%) or 0-5 amalgams (0\%).

For the subgroups that were significant in t-tests, ANOVA or Chi-square testing for trend (Tables 2 and 3) a multiple logistic regression was used to compute the age and gender adjusted odds ratios for $\mathrm{Hg}$ levels in blood or urine, or numbers of amalgams. After adjusting for age and gender, the as a dental restorative material. Dental amalgam is the end result of mixing alloy powder composed of silver( $70 \%)$, tin ( 27\%), copper $(\sim 5 \%)$ and zinc $(<1 \%)$ and liquid mercury that varies between $40 \%$ to $50 \%$ by weight. ${ }^{16}$ High-copper amalgams require less mercury in the mix. ${ }^{16}$

During placement and removal of amalgam fillings patients may become exposed to very small amounts of mercury vapour and particles. Furthermore, minute quantities of mercury vapour may leach during chewing. Emission of elemental mercury into the oral cavity is reported to be correlated with the number of amalgam surfaces (regression coefficient 0.73). ${ }^{17}$ Due to leaching mercury vapours can be inhaled and transferred to the lungs through breathing due to their presence in the ambient air and the metal can reach the gastro-intestinal tract through the saliva which carries the dissolved inorganic mercury or the abraded particles. Eley and $\operatorname{Cox}^{18}$ and Mackert and Berglund ${ }^{19}$ reviewed the consequences of mercury release from dental amalgam, its absorption accumulation and excretion by the body and ill-effects of cumulative storage. Their reviews concluded that it is unlikely that these low body fluid levels of mercury reported in the literature constitute a health hazard.

Mercury affects particularly the central nervous system and the kidney. Other reported side effects of mercury from dental amalgam include multiple sclerosis, Alzheimers disease, myalgic encephalitis and epilepsy. ${ }^{20}$ In an earlier study by Michel et al., ${ }^{21}$ no correlation was found between the total number of amalgam surfaces and fatigue, a symptom described in chronic mercury toxicity. In a subsequent study of 241 patients examined for oral galvanism, the blood mercury level averaged $17.3 \mathrm{nmol} / \mathrm{L}$ and never exceeded $50 \mathrm{nmol} / \mathrm{L}$. No renal dysfunction was uncovered in the study subjects. ${ }^{22}$ In a more recent five-year follow-up study examining neurophysiological functions of US children inconsistent differences were found in children with or without amalgam restorations. ${ }^{23}$ In an earlier report, the authors Bellinger et $a l^{24}$ did not find significant differences in adverse neuropsychological or renal effects in children whose caries were restored with dental amalgam compared to those with composite resin. A seven-year follow-up study to assess the safety of 
Table 2 Blood and urine mercury levels ( $\mathrm{nmol} / \mathrm{L}$ ) by subject groups and by amalgam fillings in mouth

\begin{tabular}{|c|c|c|c|c|c|c|c|c|}
\hline \multirow{2}{*}{ Variable } & \multirow{2}{*}{ Category } & \multirow{2}{*}{$\mathrm{n}$} & \multicolumn{3}{|l|}{ Blood } & \multicolumn{3}{|l|}{ Urine } \\
\hline & & & Mean & SD & $p$ value ${ }^{*}$ & Mean & SD & $p$ value $^{*}$ \\
\hline Total & & 56 & 19.91 & 11.80 & & 17.02 & 11.61 & \\
\hline \multirow[t]{2}{*}{ Gender } & Female & 43 & 17.97 & 10.60 & \multirow[t]{2}{*}{0.0528} & 15.69 & 11.74 & \multirow[t]{2}{*}{0.1672} \\
\hline & Male & 13 & 25.58 & 13.71 & & 21.78 & 10.35 & \\
\hline \multirow[t]{6}{*}{ Age group } & $<40$ & 11 & 14.75 & 7.40 & \multirow[t]{6}{*}{0.1042} & 15.89 & 10.98 & \multirow[t]{6}{*}{0.1974} \\
\hline & $40-44$ & 14 & 25.08 & 13.75 & & 20.91 & 12.20 & \\
\hline & $45-49$ & 5 & 12.40 & 7.37 & & 13.25 & 5.56 & \\
\hline & $50-54$ & 10 & 19.25 & 10.93 & & 13.50 & 9.43 & \\
\hline & $55-59$ & 6 & 29.25 & 13.28 & & 27.75 & 18.66 & \\
\hline & $\geq 60$ & 10 & 17.67 & 10.28 & & 11.89 & 8.30 & \\
\hline \multicolumn{9}{|l|}{ Complaints } \\
\hline \multirow[t]{2}{*}{ Oral cavity complaints } & No & 29 & 16.33 & 10.43 & \multirow[t]{2}{*}{0.0320} & 16.86 & 12.14 & \multirow[t]{2}{*}{0.9254} \\
\hline & Yes & 27 & 23.65 & 12.21 & & 17.21 & 11.29 & \\
\hline \multirow[t]{2}{*}{ Neurological } & No & 41 & 21.03 & 12.90 & \multirow[t]{2}{*}{0.3003} & 17.33 & 12.43 & \multirow[t]{2}{*}{0.7824} \\
\hline & Yes & 15 & 17.00 & 8.00 & & 16.18 & 9.47 & \\
\hline \multirow[t]{2}{*}{ Musculo-skeletal } & No & 22 & 21.16 & 13.12 & \multirow[t]{2}{*}{0.5578} & 17.93 & 11.83 & \multirow[t]{2}{*}{0.7244} \\
\hline & Yes & 34 & 19.07 & 10.99 & & 16.56 & 11.69 & \\
\hline \multirow[t]{2}{*}{ Anxiety and depression } & No & 50 & 20.64 & 12.16 & \multirow[t]{2}{*}{0.2243} & 17.53 & 11.84 & \multirow[t]{2}{*}{0.3307} \\
\hline & Yes & 6 & 13.80 & 5.85 & & 10.67 & 6.03 & \\
\hline \multicolumn{9}{|l|}{ Disorders/co-morbidities } \\
\hline \multirow[t]{2}{*}{ Allergies } & No & 30 & 17.46 & 9.09 & \multirow[t]{2}{*}{0.1469} & 17.38 & 9.75 & 0.8432 \\
\hline & Yes & 26 & 22.48 & 13.84 & & 16.65 & 13.54 & \\
\hline Auto-immune disorders & No & 48 & 19.90 & 11.65 & 0.9838 & 16.42 & 10.38 & 0.3751 \\
\hline & Yes & 8 & 20.00 & 13.61 & & 21.40 & 19.42 & \\
\hline Depression & No & 50 & 20.64 & 12.16 & 0.2243 & 17.53 & 11.84 & 0.3307 \\
\hline & Yes & 6 & 13.80 & 5.85 & & 10.67 & 6.03 & \\
\hline Multiple sclerosis & No & 54 & 19.33 & 11.22 & 0.0186 & 15.74 & 10.05 & 0.0011 \\
\hline & Yes & 2 & 47.00 & - & & 42.00 & 15.56 & \\
\hline Cardiovascular disorders & No & 43 & 20.62 & 12.37 & 0.4358 & 18.18 & 12.34 & 0.1985 \\
\hline & Yes & 13 & 17.30 & 9.49 & & 12.25 & 6.48 & \\
\hline Others & No & 43 & 21.83 & 11.55 & 0.0567 & 19.41 & 11.85 & 0.0113 \\
\hline & Yes & 13 & 14.33 & 11.18 & & 8.56 & 5.17 & \\
\hline Number of amalgams & $0-5$ & 8 & 23.75 & 17.10 & 0.4641 & 15.00 & 12.86 & 0.7724 \\
\hline & $6-10$ & 18 & 16.93 & 8.35 & & 18.14 & 10.52 & \\
\hline & $>10$ & 19 & 19.81 & 13.16 & & 18.73 & 13.19 & \\
\hline
\end{tabular}

dental amalgam restorations in children reported no significant differences in neurobehavioural assessments or in nerve conduction velocity when compared to those restored with composite resin..$^{25}$ Mortada et al. ${ }^{13}$ compared a group with amalgam restorations to a population without dental amalgam restorations. They reported a significant correlation between blood and urine $\mathrm{Hg}$ levels to the number of amalgam restorations. They also reported significantly higher urinary excretion of markers 


\begin{tabular}{|c|c|c|c|c|c|c|c|c|c|}
\hline \multirow[t]{3}{*}{ Variable } & \multirow[t]{3}{*}{ Category } & \multirow[t]{3}{*}{$\mathrm{n}$} & \multicolumn{6}{|c|}{ Number of amalgams } & \multirow[t]{3}{*}{ p value* } \\
\hline & & & \multicolumn{2}{|c|}{$0-5$} & \multicolumn{2}{|c|}{$6-10$} & \multicolumn{2}{|c|}{$>10$} & \\
\hline & & & $n$ & $\%$ & $\mathrm{n}$ & $\%$ & $\mathrm{n}$ & $\%$ & \\
\hline Total & & $45^{+}$ & 8 & 17.8 & 18 & 40.0 & 19 & 42.2 & \\
\hline \multirow[t]{2}{*}{ Gender } & Female & 34 & 5 & 62.5 & 15 & 83.3 & 14 & 73.7 & \multirow[t]{2}{*}{0.8416} \\
\hline & Male & 11 & 3 & 37.5 & 3 & 16.7 & 5 & 26.3 & \\
\hline \multirow[t]{6}{*}{ Age group } & $<40$ & 11 & 2 & 25.0 & 8 & 44.4 & 1 & 5.3 & \multirow[t]{6}{*}{0.0063} \\
\hline & $40-44$ & 11 & 3 & 37.5 & 5 & 27.8 & 3 & 15.8 & \\
\hline & $45-49$ & 5 & 1 & 12.5 & 0 & 0.0 & 4 & 21.1 & \\
\hline & $50-54$ & 6 & 1 & 12.5 & 3 & 16.7 & 2 & 10.5 & \\
\hline & $55-59$ & 4 & 0 & 0.0 & 1 & 5.6 & 3 & 15.8 & \\
\hline & $\geq 60$ & 8 & 1 & 12.5 & 1 & 5.6 & 6 & 31.6 & \\
\hline \multicolumn{10}{|l|}{ Complaints } \\
\hline \multirow[t]{2}{*}{ Oral cavity complaints } & 0 & 25 & 2 & 25.0 & 12 & 66.7 & 11 & 57.9 & \multirow[t]{2}{*}{0.3233} \\
\hline & 1 & 20 & 6 & 75.0 & 6 & 33.3 & 8 & 42.1 & \\
\hline \multirow[t]{2}{*}{ Neurological } & 0 & 33 & 8 & 100.0 & 10 & 55.6 & 15 & 78.9 & \multirow[t]{2}{*}{0.8243} \\
\hline & 1 & 12 & 0 & 0.0 & 8 & 44.4 & 4 & 21.1 & \\
\hline \multirow[t]{2}{*}{ Musculo-skeletal } & 0 & 17 & 4 & 50.0 & 4 & 22.2 & 9 & 47.4 & \multirow[t]{2}{*}{0.5951} \\
\hline & 1 & 28 & 4 & 50.0 & 14 & 77.8 & 10 & 52.6 & \\
\hline \multirow[t]{2}{*}{ Anxiety and depression } & 0 & 41 & 8 & 100.0 & 16 & 88.9 & 17 & 89.5 & \multirow[t]{2}{*}{0.5178} \\
\hline & 1 & 4 & 0 & 0.0 & 2 & 11.1 & 2 & 10.5 & \\
\hline \multicolumn{10}{|l|}{ Disorders/co-morbidities } \\
\hline \multirow[t]{2}{*}{ Allergies } & 0 & 28 & 3 & 37.5 & 13 & 72.2 & 12 & 63.2 & \multirow[t]{2}{*}{0.4630} \\
\hline & 1 & 17 & 5 & 62.5 & 5 & 27.8 & 7 & 36.8 & \\
\hline \multirow[t]{2}{*}{ Auto-immune disorders } & 0 & 39 & 8 & 100.0 & 17 & 94.4 & 14 & 73.7 & \multirow[t]{2}{*}{0.0317} \\
\hline & 1 & 6 & 0 & 0.0 & 1 & 5.6 & 5 & 26.3 & \\
\hline \multirow[t]{2}{*}{ Depression } & 0 & 41 & 8 & 100.0 & 16 & 88.9 & 17 & 89.5 & 0.5178 \\
\hline & 1 & 4 & 0 & 0.0 & 2 & 11.1 & 2 & 10.5 & \\
\hline Multiple sclerosis & 0 & 43 & 8 & 100.0 & 17 & 94.4 & 18 & 94.7 & 0.6552 \\
\hline & 1 & 2 & 0 & 0.0 & 1 & 5.6 & 1 & 5.3 & \\
\hline Cardiovascular disorders & 0 & 38 & 8 & 100.0 & 16 & 88.9 & 14 & 73.7 & 0.0675 \\
\hline & 1 & 7 & 0 & 0.0 & 2 & 11.1 & 5 & 26.3 & \\
\hline Others & 0 & 34 & 6 & 75.0 & 14 & 77.8 & 14 & 73.7 & 0.8640 \\
\hline & 1 & 11 & 2 & 25.0 & 4 & 22.2 & 5 & 26.3 & \\
\hline
\end{tabular}

of renal tubular damage (eg albumininuria and raised glutamyltransferase (GT)) in persons with amalgam restorations than without. In this study group comparison by the number of amalgam restorations failed to find any association with reported medical symptoms (Table 3).
In the case series reported by Herrstrom et al. ${ }^{22}$ psychiatric disorders were the main diagnosis for $42.7 \%$ of these patients including general anxiety and fits of panic. Forty percent of these patients were unemployed or not working because of illness. The most common age group was
40-49 years for both men and women. Feeling tired, exhaustion and muscle and joint pain are among the most common symptoms among people complaining of mercury toxicity. This profile of symptoms has been linked to resemble complaints by subjects with 'multiple chemical sensitivity 
Table 4 Risks of a complaint/disorder on biochemical values or the number of amalgams in mouth

\begin{tabular}{|c|c|c|c|c|c|c|c|c|c|}
\hline \multirow[t]{2}{*}{ Symptom } & \multicolumn{3}{|l|}{ Blood } & \multicolumn{3}{|l|}{ Urine } & \multicolumn{3}{|c|}{ Number of amalgams (>10 vs $0-10$ ) } \\
\hline & Adjusted $\mathrm{OR}^{*}$ & $95 \% \mathrm{Cl}$ & $\mathrm{p}$ value & Adjusted $\mathrm{OR}^{*}$ & $95 \% \mathrm{Cl}$ & $\mathrm{p}$ value & Adjusted $\mathrm{OR}^{*}$ & $95 \% \mathrm{Cl}$ & $\mathrm{p}$ value \\
\hline Oral complaints & 1.074 & $(1.005,1.149)$ & 0.0362 & 1.01 & $(0.953,1.069)$ & 0.7451 & 0.501 & $(0.122,2.055)$ & 0.3372 \\
\hline Auto-immune disorders & 1.006 & $(0.927,1.091)$ & 0.8929 & 1.046 & $(0.957,1.142)$ & 0.3209 & 5.031 & $(0.469,53.944)$ & 0.1819 \\
\hline Multiple sclerosis & 2.551 & $\begin{array}{l}(0.076 \\
85.205)\end{array}$ & 0.6009 & 1.309 & $(0.925,1.851)$ & 0.1282 & 1.835 & $(0.072,47.003)$ & 0.7137 \\
\hline Others & 0.941 & $(0.874,1.013)$ & 0.1043 & 0.823 & $(0.696,0.974)$ & 0.0233 & 1.032 & $(0.222,4.786)$ & 0.9681 \\
\hline
\end{tabular}

syndrome.26 Among 465 patients attending a general practice in New Zealand complaining of having chronic mercury toxicity severe fatigue (32\%), memory loss (89\%) and depression (28\%) were reported..$^{27}$

Limited evidence exists for an association of chronic mercury exposure due to dental amalgams with multiple sclerosis (MS).$^{28}$ Siberud ${ }^{29}$ reported a poorer mental health status in a cohort of MS patients with amalgam fillings compared to those without, and attributed the difference to mercury toxicity from dental amalgam. A more recent study from Canada reported higher number of amalgams and earlier age of amalgam restoration placements in subjects with $\mathrm{MS}^{30}$ but in comparison with a control group these differences were not significant.

This study confirms the conclusions by previous authors as blood and urine levels were found to be within the normal range in the cohort described here. Two patients with MS in this study had raised Hg levels (Table 2) compared with the rest of group who did not report MS, although their blood levels were still within the expected normal range $(<50 \mathrm{nmol} / \mathrm{L})$. In the logistic regression however, this association did not amount to an increased risk $(\mathrm{OR}=2.55, \mathrm{CI}=0.07-85.2)$ probably due to the small sample size. The findings could also be explained as due to negative confounding by better access to NHS dental care for the MS group or a high caries incidence (unrelated to MS) as reported by McGrother et al. ${ }^{31}$

The study group in our report shared most of the physical symptoms and demographic features reported in earlier studies. ${ }^{11}$ Even in this small sample we could infer that somatisation of perceived symptoms was a common finding.
Because of concerns over adverse effects of mercury, some patients with alleged adverse effects may request the removal of their amalgam restorations. This study provides evidence that biochemical assessment of body fluids will allow both the clinician and the patient to make a rational judgement as to the necessity to remove amalgam fillings. In this series none were advised to have their metallic fillings replaced. For people with some perceived disorders biochemical validation provides objective assessment and reassurance. This may help them to accept the fact that amalgam fillings are not the cause of their symptoms rather than resorting to blind restorative interventions.

The issue of dental amalgam as causing ill health remains controversial. Genetic variations in mercury metabolism may explain some inconsistent findings in clinical trials. ${ }^{27}$ Reported studies show little evidence of effects from dental amalgam on chronic disease incidence or mortality. This audit of people complaining of chronic symptoms associated with amalgam restorations found mercury levels in blood or urine to be within normal laboratory range. Their perceived symptoms may be psychological but we did not include any measurements. Further studies on this aspect may be helpful in their management. The evidence from this study does not support some lay people's view that exposure to mercury from amalgam may be a factor in the development of some chronic disorders. The questionable association noted with MS was not confirmed in the logistic regression analysis and needs further study.

\footnotetext{
1. Jones D W. Putting dental mercury pollution into perspective. Br Dent J 2004; 197: 175-177.

2. Magos L, Clarkson T W. Overview of the clinical toxicity of mercury. Ann Clin Biochem 2006;
} 43: 257-268.
3. Harvey J, Harwell L, Summers J K. Contaminant concentrations in whole-body fish and shellfish from US estuaries. Environ Monit Assess 2008: 137: 403-412.

4. Kales S N, Goldman R H. Mercury exposure: current concepts, controversies and a clinic's experience. J Occup Environ Med 2002; 44: 143-154.

5. Woods J S, Martin M D, Leroux B G et al. The contribution of dental amalgam to urinary mercury excretion in children. Environ Health Perspect 2007; 115: 1527-1531.

6. ADA Council on Scientific Affairs. Dental amalgam: update on safety concerns. J Am Dent Assoc 1998; 129: $494-503$.

7. Yip H K. Dental amalgam and human health Int Dent J 2003: 53: 464-468.

8. Osborne J W, Albino J E. Psychological and medical effects of mercury intake from dental amalgam. A status report for the American Journal of Dentistry. Am J Dent 1999; 12: 151-156.

9. Hörsted-Bindslev P. Amalgam toxicityenvironmental and occupational hazards. J Dent 2004; 32: 359-365.

10. Lindbohm M L, Ylostalo P, Sallmen M et al. Occupational exposure in dentistry and miscarriage. Occup Environ Med 2007; 64: 127-133.

11. Bailer J, Rist F, Rudolf A, Staehle et al. Adverse health effects related to mercury exposure from dental amalgam fillings: toxicological or psychological causes? Psychol Med 2001; 31: 255-263.

12. Tchounwo P B, Ayensu W K, Ninashvili N, Sutton D. Environmental exposure to mercury and its toxicopathologic implications for public health. Environ Toxicol 2003; 18: 149-175.

13. Mortada W I, Sobh M A, El-Defrawy M M, Farahat $\mathrm{S}$ E. Mercury in dental restoration: is there a risk of nephrotoxicity? J Nephrol 2002; 15: 171-176.

14. Clifton J C. Mercury exposure and public health. Pediatr Clin North Am 2007; 54: 237-269.

15. Mutter J, Naumann J, Schneider R, Walach H, Haley B. Mercury and autism: accelerating evidence? Neuro Endocrino Lett 2005; 26: 439-446.

16. Materials in Dentistry. Principals and Applications. J. F. Ferrance. Philadelphia; Lippincott, Williams \& Wilkins 2001, p121.

17. Skare I, Engqvist A. Human exposure to mercury and silver released from dental amalgam restorations. Arch Environ Health, 1994; 49: 384-394.

18. Eley B M, Cox S W. The release, absorption and possible health effects of mercury from dental amalgam: a review of recent findings. Br Dent J 1993; 175: 355-362.

19. Mackert J R, Berglund A. Mercury exposure from dental amalgam fillings: absorbed dose and the potential for adverse health effects. Crit Rev Oral Biol Med 1997; 8: 410-436.

20. Clarkson T W, Magos L, Meyers G J. The toxicology of mercury - current exposures and clinical manifestations. N Eng/ J Med 2003; 349: 1731-1737.

21. Michel I, Norback D, Edling C. An epidemiologic study of the relation between symptoms of fatigue, dental amalgam and other factors. Swed Dent J 1989; 13: 33-38.

22. Herrstrom $P$, Hogstedt B. Clinical study of oral galvanism: no evidence of toxic mercury exposure 
but anxiety disorders an important background factor. Scand J Dent Res 1993; 101: 232-237.

23. Bellinger D C, Daniel D, Trachtenberg F, Tavares M, McKinlay S. Dental amalgam restorations and children's neuropsychological function: the New England Children's amalgam trial. Environ Health Perspect 2007; 115: 440-446.

24. Bellinger D C, Trachtenberg F, Barregard L et al. Neuropsychological and renal effects of dental amalgam in children - a randomised clinical trial. JAMA 2006; 295, : 1775-1783.

25. DeRouen T A, Marin M D, Leroux B G et al. Neurobehavioural effects of dental amalgam in children - A randomised clinical trial. JAMA 2006; 295,: 1784-1792.

26. Malt U F, Nerdrum P, Oppedal B et al. Physical and mental problems attributed to dental amalgam fillings. Psychosom Med 1997; 59: 32-41.

27. Wojcik D P, Godfrey M E, Christie D, Haley B E. Mercury toxicity as chronic fatigue, memory impairment and depression: diagnosis, treatment, susceptibility, and outcome in a New Zealand general practice setting (1994-2006). Neuro Endocrinol Lett 2006; 27: 415-423.

28. Bates M N. Mercury amalgam dental fillings: an epidemiologic assessment. Int J Hygiene Environ
Health 2006; 209: 309-316.

29. Siblerud R L. A comparison of mental health of multiple sclerosis patients with silver/mercury dental fillings and those with fillings removed. Psychol Rep 1992; 70: 1139-1151.

30. Bangsi D, Ghadirian P, Ducic S, et al. Dental amalgam and multiple sclerosis: a case-control study in Montreal, Canada. Int J Epidemiol 1998; 27: 667-671.

31. McGrother C W, Dugmore C, Phillips M J, Raymond N T, Garrick P, Baird W O. Multiple sclerosis, dental caries and amalgam fillings - a case control study Br Dent J 1999; 187: 261-264. 